\title{
La educación y la conservación de la naturaleza: Una alianza impostergable
}

\section{Education and Nature Conservation: an Urgent Alliance}

\author{
Ana Isabel Pereira Pérez. \\ Sede de Guanacaste \\ Universidad de Costa Rica \\ Guanacaste, Costa Rica \\ pereiraanaisabel@yahoo.com \\ Nuria Mairena Rodríguez ${ }^{2}$ \\ Sede de Guanacaste \\ Universidad de Costa Rica \\ Guanacaste, Costa Rica \\ nurimai@gmail.com
}

Recibido 07 de noviembre de 2010 • Aceptado 09 de marzo de 2011

Resumen. El presente ensayo tiene como propósito señalar la riqueza que posee Costa Rica en cuanto a flora y fauna se refiere y el riesgo que se corre, si no somos capaces de formar a los ciudadanos para su apropiada conservación. En ese sentido, las proponentes argumentan que, mediante la educación, se puede llegar a promover en los ciudadanos y las comunidades, la conservación de la naturaleza, aspecto que se constituye en una necesidad, de ahí que se plantee que la conservación y la educación han de constituir una alianza impostergable.

Palabras claves. Riqueza biológica, aula abierta, conservación, educación, áreas naturales, comunidades, naturaleza.

Abstract. This paper aims at pointing out not only Costa Rica's richness in terms of flora and fauna, but also the risk we face if we are unable to educate citizens for the appropriate nature conservation. In this sense, the authors state that it is through education that nature conversation, which is a necessity, can be promoted among citizens and communities. This is the reason why conservation and education must create an urgent alliance.

Keywords. Biological richness, open classroom, conservation, education, natural areas, communities, nature.

1 Máster en Ornitología de la Universidad de Costa Rica. Bachiller en Biología de la Universidad de Costa Rica. Coordinadora de la Iniciativa Cultura Ambiental en Acción, UCR-CONARE-Región Chorotega. Laboró como profesora universitaria en la carrera de Biología de la Universidad de Costa Rica, en la Sede Central y actualmente forma parte del equipo de docentes de la carrera de Turismo Ecológico en la Sede Guanacaste. Experiencia como investigadora en el área de la ornitología. Experiencia en asesorías de impacto ambiental. Posee artículos publicados en las áreas de ecología de alimentación de aves limícolas, dispersión de semillas por aves y camuflaje de nidos de aves.

2 Graduada en la UCR como Magistra en Educación, énfasis en Administración Educativa, Licenciada en Curriculum y Bachiller en Educación Graduada en la UCR como Magistra en Educación, énfasis en Administración Educativa, Licenciada en Curriculum y Bachiller en Educación Preescolar. Docente Universitaria para las carreras de Educación Preescolar, Primaria, Preescolar y Primaria con énfasis en Inglés. Coordinadora de las carreras de Bachillerato y Licenciatura en Educación Preescolar en la Sede de Guanacaste, UCR. Directora y lectora en tesis de grado en la Universidad de Costa Rica, en Educación. Responsable de proyectos de investigación y acción social. Laboró como Tutora del Posgrado en Gestión y Liderazgo de la Universidad Nacional, región Chorotega. Responsable y colaboradora en Iniciativas CONARE, CRI Chorotega Cultura Ambiental en Acción y Centro de Intervención Pedagógica: Dejando Huellas. 


\section{Introducción}

Costa Rica es un país caracterizado por su gran riqueza biológica, y su diversidad es notable en cuanto a ecosistemas. Se encuentran, en él, importantes áreas de bosque tropical húmedo y muy húmedo, sistemas de humedales de importancia mundial, bosques nubosos, páramos y bosque seco tropical restringido al pacífico norte de Costa Rica, entre otros (Hartshorn, 1991;Valerio, 1991).

No obstante esta diversidad, algunas zonas de nuestro país se caracterizan por su vulnerabilidad, lo cual hace necesario que los ciudadanos tomemos conciencia de ello y nos aboquemos a la búsqueda de alternativas para su conservación, papel que bien puede ser fortalecido mediante estrategias pedagógicas.

En el caso de Guanacaste, existe, por ejemplo, una de las zonas de vida más frágil y menos protegida del mundo: el bosque seco (Sánchez-Azofeifa et al., 2003), el cual ha sido básicamente explotado por la ganadería, debido a sus condiciones climáticas. Estas hacen necesario fortalecer y conservar algunas de las zonas o áreas naturales de nuestro territorio. En ese sentido, se cree, con firmeza, que la educación puede jugar un papel preponderante en la aplicación de alternativas y estrategias que, mediante la formación de los niños y las niñas, jóvenes y adultos, puedan promover valores conservacionistas que lleven al cuidado del medio ambiente.

Respecto de lo anterior, se propone el establecimiento de alianzas estratégicas entre la educación y la conservación del ambiente. Para lograr dichas alianzas se pueden utilizar variadas estrategias, no obstante lo dicho, creemos importante que las mismas retomen la incorporación de experiencias concretas en el entorno natural. Por ello, el presente ensayo busca evidenciar la necesidad de plantear dichas alianzas, mediante la estrategia del aula abierta, con el fin de facilitar y promover la conservación de los entornos naturales.

\section{Desarrollo}

A pesar de la gran importancia que, para el desarrollo sustentable tiene la conservación de nuestros ambientes naturales, Costa Rica ha venido sufriendo un deterioro ambiental a nivel nacional. A partir de 1950, con la expansión de los cultivos de café (Pérez, 1977), los ecosistemas inician su transformación en agro ecosistemas, con sus consecuencias negativas sobre la biodiversidad, incrementadas con el uso de agroquímicos, como fertilizantes y plaguicidas (Quesada, 2008). Posteriormente, se inicia el cultivo del banano, con lo cual se eliminan grandes extensiones de bosques húmedos del Caribe y de la vertiente del Pacífico. Además se expanden las actividades ganaderas, causantes de la destrucción de más del 60\% de la cobertura boscosa del país, se elimina, así, el hábitat de cientos de especies de animales y plantas y se generan graves problemas de erosión del suelo (Orozco, 1996; Quesada, 2008). Para 1940, se estimaba una cubierta boscosa de entre 68-78\%, mientras que en los 90 apenas alcanza un 30\% (Orozco, 1996; Rosero-Bixby y Palloni, 1998).

Estos y otros sistemas productivos desarrollados en el país, asociados al crecimiento poblacional, están relacionados con diferentes problemas de degradación del medio ambiente, tales como uso inadecuado del suelo, deterioro de las cuencas hidrográficas, contaminación del agua, mal manejo de aguas negras y desechos, entre otros. Todos ellos repercuten 
directamente en la sociedad, la economía y, por supuesto, en el ambiente, socavando la estabilidad productiva del país (Orozco, 1996).

Específicamente, en la provincia de Guanacaste, la actividad agropecuaria ha tenido un efecto negativo sobre la cubierta boscosa, pues se han eliminado grandes extensiones de bosque, para permitir el cultivo de productos como el arroz y la caña de azúcar, pero también, la crianza de ganado de carne y leche (Cárdenas, Harvey, Ibrahim y Finegan, 2003). Según Palma (2005), se han realizado diversos estudios en el trópico latinoamericano, en relación con el uso de árboles y arbustos en los sistemas ganaderos, en los cuales se considera de vital importancia "(...) reconsiderar la relación de la ganadería bovina con el uso de la tierra, la deforestación, el empleo rural y la biodiversidad, para desarrollar alternativas que permitan la transformación de la ganadería actual en un sistema sostenible, menos incompatible con la diversidad biológica y más apropiada para lograr el bienestar humano". (p. 2) Cualquier estrategia, que logre revertir al menos en una parte, el impacto que nuestras tierras guanacastecas han sufrido gracias no solamente al uso en la ganadería, sino también en la agricultura, es muy beneficioso, por lo que las actividades silvopastoriles pueden ser una alternativa que incremente la biodiversidad (Cárdenas et al. 2003).

En las zonas rurales del país, aproximadamente el 63\% del agua para consumo humano proviene de aguas subterráneas (Abarca, 2001), de ahí la importancia de los acuíferos en la región guanacasteca. Los mantos acuíferos son muy frágiles en regiones como esta (Arellano y Vargas, 2001), donde existe un periodo de fuerte disminución de las precipitaciones, pues estos se recargan con el agua de lluvia (Abarca, 2001). Resulta preocupante el hecho de que la mayoría de las cuencas hidrográficas presentan diversos grados de deterioro ambiental, lo cual reduce la captación de agua necesaria para el consumo humano (Merayo, 2004). Sin embargo, en algunas cuencas, como es el caso de Morote, se ha venido mejorando el uso del suelo, pues la actividad ganadera ha ido disminuyendo, se han empezado a llevar a cabo actividades de reforestación con fines comerciales y algunos terrenos han sido abandonados para su regeneración natural (Hernández y Zúñiga, 2005).

El incremento tan acelerado de la actividad turística en la región de Guanacaste y la mala planificación del desarrollo inmobiliario, asociado a dicha actividad, ha generado serios problemas ambientales (Argos, s. f.; Román, 2007). En lo que al uso del agua se refiere, en algunas comunidades costeras los acuíferos han sido tan explotados que se han salinizado, haciéndolos inútiles para el consumo humano y para actividades agrícolas y de otra índole; este ha sido el caso de playa Flamingo, donde se tuvieron que cerrar los pozos que lo abastecían de agua y de Playa Grande, donde cuatro de los ocho pozos mostraron intrusión salina (Arellano y Vargas, 2001).

En un estudio realizado por Vargas (s. f. a), en relación con los impactos ambientales de los desarrollos turísticos y residenciales del pacífico norte, se menciona que “(...) la costa del Pacífico de Costa Rica no está preparada para responder a las crecientes necesidades de infraestructura para el abastecimiento de agua potable o para la disposición adecuada de aguas residuales" (p. 42); que los acueductos locales no pueden cubrir las necesidades locales y que los acuíferos podrían verse afectados negativamente. Ejemplo de estos conflictos lo constituye el caso de la comunidad de Sardinal, la cual se ha opuesto a la ampliación de su acueducto hacia la costa, para dar abasto a las poblaciones costeras y desarrollos turísticos y residenciales, por temor al colapso de su acueducto (Arias et al. 2009). Playa Hermosa, Playa Panamá, El Coco y Ocotal, son ejemplo de comunidades costeras cuyos acuíferos están en riesgo (Vargas, s. f. b). 
Asociado a estas actividades turísticas, se agrega el problema del manejo de las aguas residuales y aguas negras, provenientes de los grandes complejos hoteleros desarrollados en las zonas costeras, como el complejo Papagayo y del desarrollo inmobiliario y residencial de playas como Tamarindo y Sámara, por ejemplo (Román, 2007; Vargas, s. f. a). A pesar de que dentro de los requerimientos para su funcionamiento está el establecimiento de plantas de tratamiento de aguas, en ocasiones la visitación es tan alta que dichas plantas no dan abasto, como sucedió con el hotel Allegro Papagayo, que colocó tuberías clandestinas para eliminar al ambiente los desechos de este tipo, pues la planta colapsó. Dicho hotel tuvo que cerrar sus puertas hasta construir una nueva planta de tratamiento capaz de manejar la cantidad de residuos producidos (Vargas, s. f. a).

En Sámara, el manejo de las aguas negras también ha puesto en jaque a la comunidad, pues las filtraciones de los tanques sépticos contaminaron el agua de mar; lo mismo se ha reportado para Tamarindo, en cuyas aguas se han registrado niveles peligrosos de coliformes fecales (Vargas, s. f. a). En esta última comunidad, no existen plantas de tratamiento de agua, o las plantas existentes no trabajan adecuadamente; además no ha logrado mantener el galardón de la Bandera Azul Ecológica, pues perdió todos los puntos relacionados con el manejo de las aguas residuales entre otros (Vargas, s. f. a).

Otro problema ambiental, generado por el crecimiento poblacional, el cambio en el uso de la tierra, la construcción de hoteles y marinas, producto del incremento en la actividad turística, especialmente en las zonas costeras, es el deterioro de la vegetación costera, en concreto, los manglares, uno de los ecosistemas más amenazados del mundo (Jiménez, 2004; Zamora-Trejos y Cortés, 2009). Por otra parte, Román (2007) comenta algunos impactos ecológicos en la zona costera, como es el caso del Estero Icacal, en donde se ha adicionado material de lastre para hacer una calle de acceso a una propiedad privada, con la eventual interrupción del flujo de agua, tan importante para el ecosistema; en Playa Pargos hay violación a la ley de la Zona Marítimo Terrestre; estero Platanar fue alterado con material de relleno; Playa Blanca, Junquillal presenta material de relleno y una cerca de postes de cemento dentro del manglar. En Playa Matapalo, se han denunciado, por parte de los vecinos y organizaciones ecologistas, actividades ilegales realizadas por parte de empleados del Hotel Riu y han actuado organizadamente en un intento por revertir al menos parcialmente el daño hecho al manglar aleñado al hotel (Vargas, 2009 b). Igualmente, en Playa Caletas, el programa de Restauración de Tortugas Marinas (PRETOMA) ha venido denunciando anormalidades en las zonas de manglar del Refugio Nacional de Vida Silvestre Caletas Arío, generadas por Agropecuaria Caletas S.A., los que, al parecer, han esparcido venenos causante de la muerte masiva de peces, lo que ha puesto en riesgo una de las principales playas de anidación de cuatro especies de tortugas marinas, incluida la Baula, especie en peligro crítico de extinción (El País.cr, 2010).

En un estudio realizado por Bonilla-Carrion y Rosero-Bixby, 2004, para estimar la presión demográfica sobre los bosques y áreas protegidas del país, se encontró que en Costa Rica la gran mayoría de sus habitantes vive a menos de $20 \mathrm{~km}$ del bosque. Guanacaste, Hojancha, Nicoya y Nandayure son los cantones cuya población se encuentra en mayor contacto con el bosque, aunque no los que tienen mayor proporción de su territorio en bosques. Además se halló que más de la mitad de los bosques actuales de los cantones guanacastecos de Abangares, Nandayure, Hojancha, Carrillo, Nicoya y Liberia se identifican con grandes probabilidades de ser deforestados en los próximos años. La Región Chorotega ocupa el segundo lugar en porcentaje de bosque en riesgo de 
deforestación con estrés, seguida del área metropolitana. En el caso específico de Santa Cruz, estos autores plantearon que en el año 2000 poseía un $43 \%$ de su territorio cubierto por bosque, pero el $50 \%$ de él se encontraba en riesgo.

La cercanía de la población humana a las áreas boscosas del país ha generado una fuerte presión, no solo en lo que a deforestación se refiere, sino también por la caza, la extracción de madera, plantas de uso medicinal y ornamental, etc. Esta proximidad del ser humano a áreas boscosas no ha redundado en un mejor conocimiento de los procesos biológicos, por lo que no se puede asociar a una buena comprensión de aspectos de conservación, por parte de los pobladores. Es fundamental, entonces, generar un cambio de la cultura ambiental desarrollada a lo largo del tiempo.

En la actualidad, se ha ido perdiendo el contacto con la naturaleza. Esta se encuentra cada vez más lejos del círculo de actividades humanas, pues se ha ido restringiendo a pequeños fragmentos de bosque lejos de los centros urbanos. En esta época, los niños conocen la naturaleza a través de la televisión o la Internet, pero no experimentan lo que es convivir realmente con ella. Por ello, es imperativo el trabajo en un verdadero cambio de la cultura ambiental, si se quiere tener un desarrollo sustentable, no solo a nivel local, sino regional. Este cambio debe iniciar desde la infancia, por lo que es fundamental el trabajo en la educación ambiental desde los primeros niveles educativos. Por ello, uno de los objetivos de la Iniciativa Cultura Ambiental en Acción, proyecto de Regionalización Interuniversitario apoyado por CONARE y en el cual participan profesionales de la Universidad de Costa Rica, la Universidad Nacional y el Instituto Tecnológico de Costa Rica, es fomentar el uso del aula abierta en niños y niñas de educación preescolar y primaria, de tal forma que puedan palpar la naturaleza, comprender su funcionamiento y convertirse en verdaderos defensores de la misma.

En este sentido, la educación ambiental es el resultado de una reorientación y articulación de las diversas disciplinas y experiencias educativas que faciliten la percepción integrada del medio ambiente, haciendo posible una acción más racional y capaz de responder de manera eficiente a las necesidades actuales en materia de conservación.

Según Guillén (1996, p. 107):

Las líneas de acción de la Educación Ambiental son muy diversas: se asume que deben propiciar estrategias preventivas y reorientar patrones de consumo, así como promover la corresponsabilidad y la participación social. En estos procesos se propone la formación de individuos que puedan modificar sus sistemas de valores y que a su vez se inserten en un esquema social de relaciones más solidarias, cooperativas, autónomas y equitativas (este es un buen momento para distinguir la equidad en términos de reconocimiento de relaciones de desigualdad que deben promover un trato diferenciado de estos desiguales). La tolerancia, la pluralidad y el compromiso social son algunos de los valores esenciales que se deberían promover.

En ese mismo sentido, también Fernández y Fallas (2007) reafirman la importancia de la educación ambiental como un instrumento para mejorar la relación del ser humano con el medio ambiente, a través de la educación formal y no formal. Tal relación permitirá la formación de actitudes y aptitudes tendientes a promover la sensibilización a través de las acciones educativas. Con ella se pretende dar respuesta a la problemática ambiental, enlazando la realidad del entorno, la percepción ambiental de las comunidades educativas, el manejo de los recursos naturales y el 
desarrollo sustentable. Desde esta perspectiva, cobran gran importancia los espacios naturales, cercanos a las áreas urbanas, que sirvan como espacios formativos en este campo.

Para Pérez, Ramírez, Monroy y Campos (2006, p. 8):

La educación ambiental es un proceso continuo y permanente donde deben participar diferentes actores sociales, representa una estrategia para solucionar los problemas ambientales de las comunidades, regiones o países, ya que tiene efectos de carácter multiplicador y debe fundamentarse en promover acciones efectivas de cultura ambiental, que fomenten la participación de la población y ayuden a evitar, disminuir o solucionar los problemas del entorno inmediato de las sociedades. No es recomendable la enseñanza de conceptos ecológicos, geográficos, ambientales y económicos, sino, más bien, promover una cultura para el ambiente.

Por otra parte, la flexibilización curricular participativa se orienta hacia la planificación congruente con los planteamientos de la Política Educativa Nacional, pero con un enfoque innovador, en donde los participantes exploren, investiguen, descubran, consulten y desarrollen capacidades de gestión ambiental.

Como se ha mencionado, en esa búsqueda de promover la participación de las opciones curriculares, es posible plantear sugerencias acerca de variadas opciones mediante las cuales se puedan establecer alianzas con las instituciones educativas del país en procura de buscar la formación de ciudadanos más responsables con su entorno, deseosos de mantener las áreas naturales y de fomentar valores conservacionistas en las futuras generaciones.

En cuanto a lo señalado, las autoras plantean una propuesta integrada mediante la estrategia del aula abierta en áreas naturales, donde sea posible la inserción no solo de la comunidad, sino también, de las instituciones educativas en la búsqueda de la protección de las zonas que se ubiquen cerca de las distintas comunidades. El conocer dichas zonas, el acercarse a ellas, el promover la formación del estudiantado no únicamente desde los contenidos programáticos, sino también desde las vivencias concretas con la naturaleza, se constituye en una excelente herramienta para la vinculación entre educación y naturaleza, es justo ahí, donde se hace impostergable esa alianza en búsqueda de la conservación del ambiente y el compromiso de todos y todas.

El aula abierta, como estrategia, permite ubicar una zona o área natural cercana a una comunidad y, desde ella, es posible involucrar no solo a los ciudadanos, sino también a docentes y a estudiantes, para que, de manera coordinada, ejecuten acciones que permitan tomar esas áreas como fuentes de conocimientos, como enlaces entre los contenidos programáticos y la naturaleza misma.

Para el diseño y aplicación de estrategias de trabajo mediante aula abierta, es posible considerar, para su enriquecimiento, aspectos como los que a continuación se señalan:

- Analizar las condiciones específicas que tienen, las distintas comunidades educativas o regiones del país.

- Identificar en cada área específica, los problemas ecológicos, ambientales y forestales que impactan el ambiente y la comunidad. 
- Enfocar la educación ambiental desde una perspectiva holística.

- Respetar la diversidad sociocultural de las regiones e instituciones que participen de la experiencia de aula abierta.

- Comprender que cada persona es miembro de una comunidad de partes interdependientes.

- Diseñar y aplicar actividades de sensibilización ambiental, concientización ecológica, valoración del ambiente y legislación ambiental.

- Priorizar en el diseño y aplicación de actividades prácticas y de experiencia, mediante las cuales, grupos estudiantiles, padres y madres de familia, y miembros de la comunidad en general, puedan acercarse a las áreas naturales y aprender en ellas.

- Educar para la responsabilidad.

- Crear una conciencia ambiental, promoviendo que cada persona se sienta parte de la naturaleza, valorando el hecho de que cada comunidad humana incluye también el suelo, el aire, el agua, las plantas y los animales.

- Desarrollar valores de solidaridad y respeto por el ambiente.

- Reflexionar acerca de las consecuencias de nuestras acciones y estilos de vida sobre el entorno.

- Reforzar la idea de que toda especie tiene derecho a la supervivencia continuada en el ambiente en el que vive.

- Enfatizar que nuestro bienestar actual no debe prevalecer sobre el de las generaciones futuras.

Desde la perspectiva planteada, la educación se constituye en el elemento primordial para la formación de ciudadanos y ciudadanas preocupadas por su entorno, deseosos de proteger y conservar la naturaleza y preocupados, también, por la formación de generaciones futuras para quienes se desea el gozo y el disfrute de lo que la naturaleza ha puesto al alcance de la humanidad. Es así como se visualiza, mediante una estrategia concreta, la alianza educativa para la preservación del ambiente.

\section{Consideraciones finales}

Se ha puesto de manifiesto, aunque de manera muy general, la importancia que para el país tiene su diversidad biológica y la oportunidad que esta otorga a los ciudadanos, no solo para disfrutarla, sino también, para ejecutar acciones concretas para su preservación.

Se ha planteado, de igual manera, que existen diferentes estrategias u opciones para enlazar la educación con la conservación de la naturaleza, pero en el caso concreto de este aporte, se propone una estrategia específica: el aula abierta. Estrategia que permite un trabajo responsable por parte de las comunidades y de las instituciones educativas, no solo para identificar áreas naturales diversas, necesarias de proteger, sino también para desarrollar experiencias de aprendizaje significativas, que redunden en la formación de valores conservacionistas y en el desarrollo de hábitos y estrategias en procura de la defensa de los entornos naturales. En ese sentido, la alianza entre educación y naturaleza es, como se ha planteado previamente, impostergable; a través de la educación se deben inculcar valores de respeto, aprecio y compromiso hacia el medio ambiente, que permitan una verdadera interrelación entre el ser humano y la naturaleza, de manera que se consolide una cultura ambiental saludable. 
Ha de considerarse, además, que el Planeta es una responsabilidad de todos y todas, no es un asunto que se pueda o se deba dejar a las siguientes generaciones. Amerita una labor y compromiso para hoy. Si las generaciones actuales han podido disfrutar de la naturaleza, ello ha sido por las acciones que los antepasados realizaron.

De este modo, corresponde, ahora, colaborar y responsabilizarse de que la huella ecológica dejada por la generación de hoy no implique la pérdida de los entornos naturales, sino más bien, que las alianzas que, mediante la educación, se puedan establecer, con el cuidado de la naturaleza, y globalmente del planeta mismo, sean un objetivo común de cada uno de los seres que lo habita.

Se ha destacado, en este ensayo, la belleza y diversidad natural que tiene Costa Rica, pero de igual manera, se ha señalado que mucha de esa riqueza está en riesgo, de manera que el mejor aliado para su conservación se identifica en la educación, mediante una reorientación y articulación de disciplinas y experiencias educativas que puedan facilitar y promover valores y una percepción del medio ambiente más integrada, donde mediante acciones racionales se pueda defender la naturaleza, cuidando sus mantos acuíferos, las cuencas hidrográficas, velar por un desarrollo urbanístico y turístico respetuoso de la naturaleza y donde los niños y niñas desde los hogares y mediante el apoyo educativo, se conviertan en verdaderos guardianes del tesoro natural. Aspecto que, a criterio de quienes escriben este texto, es impostergable, dado que todos debemos compartir un mismo objetivo: la valoración de la naturaleza y su cuidado.

\section{Referencias bibliográficas}

Abarca, J. A. (2001). Sistemas integrados de tratamiento y uso de aguas residuales en América Latina: Realidad y potencial. Estudio general del caso Puntarenas, Costa Rica (Proyecto regional). Recuperado de http://bvs.per.paho.org/bvsaar/e/proyecto/generales/ casos/puntarenas.pdf

Arellano, F. y Vargas, A. (2001). Casos de contaminación por intrusión salina en acuíferos costeros de la península de Nicoya (Costa Rica). Revista Geológica de América Central 25, 77-84.

Argos, T. (s. f.). Dinámica territorial del desarrollo turístico costero: Unidad turística Guanacaste Norte. Caracterización de la Unidad Turística Guanacaste Norte, incluyendo aspectos históricos, físicos, ambientales y socioeconómicos, así como su dinámica turística. (Informe final, Cap. II). San José, Costa Rica: Crest. Recuperado de http://www.responsibletravel.org/ resources/documents/Coastal-tourism-documents/II-GUANACASTE NORTE\%5B1\%5D.pdf

Arias, M., Sagot, Á., Boeglin, N., Cortés, A., Morales, M., Fournier, M. ... Monestel, H. (2009). Implicaciones del proyecto de ampliación del acueducto El Coco-Ocotal, Guanacaste, conocido "como sardinal" (Informe final). Universidad de Costa Rica, Consejo Univeritario. Recuperado de http://www.cu.ucr.ac.cr/documen/informeFinalSardinal.pdf

Bonilla-Carrión, R. y Rosero-Bixby, L . R. (2004). Presión demográfica sobre los bosques y áreas protegidas, Costa Rica 2000. En L. Rosero-Bixby (Ed.). Costa Rica a la luz del censo del 
2000 (Proyecto Estado de la Nación e Instituto Nacional de Estadística y Censo [INEC]), (pp. 575-594). San José, C. R.: Imprenta Nacional.

Cárdenas, G., Harvey, C. A., Ibrahim, M. y Finegan, B. (2003). Diversidad y riqueza de aves en diferentes hábitats en un paisaje fragmentado en Cañas, Costa Rica. Agroforestería em las Américas 10(39-40), 78-85.

El País.cr (6 de julio de 2010). Compañía agrícola envenena refugio de tortugas, hostiga voluntarios de Pretoma. El país.cr Recuperado de http://www.elpais.cr/articulos.php?id=29882

Fernández, M. R. y Fallas, Y. (agosto, 2007). Educación ambiental: Cómo elaborar un programa de actividades ambientales para el centro educativo. IX Congreso Nacional de Ciencias. Instituto Tecnológico de Costa Rica, Cartago, Costa Rica. Recuperado de http://cientec.or.cr/ exploraciones/ ponencias2007/RocioFernandez-Yessenia Fallas.pdf

Guillén, F. C. (1996). Educación, medio ambiente y desarrollo sostenible. Revista Iberoamericana de Educación, 11, 103-110. Recuperado de http://www.oei.es/oeivirt/rie11a03.pdf

Hartshorn, G. S. (1991). Plantas. En D. Janzen (ed.), Historia Natural de Costa Rica (pp: 119-186). San José, Costa Rica: EUCR.

Hernández, E. y Zúniga, I. (setiembre, 2005). La cuenca hidrográfica como unidad alternativa para el desarrollo turístico. El caso de la cuenca del Río Morote. Guanacaste. Costa Rica. En Seminario Regional "Turismo Alternativo en la Región Centroamericana: Experiencias y acciones conjuntas para un desarrollo local de las comunidades". Sede Regional de Chorotega, Costa Rica. Recuperado de http://files.riomorote.webnode.es/20000022535ee036e84/Cuenca\%20 Morote\%20y\%20Turismo Igor\%20Zu\%C3\%B1iga \%202006.pdf

Jiménez, J. A. (2004). Mangrove forests under dry seasonal climates in Costa Rica. En G. W. Frankie, A. Mata y S. B. Vinson (eds.). Biodiversity Conservation in Costa Rica: Learning the Lesson in a Seasonal Dry Forest (pp. 136-146). Berkely, California: Univ. California Press.

Merayo, O. (noviembre, 2004). Valoración económica del agua potable en la cuenca del río Endemedio, Santa Cruz, Guanacaste, Costa Rica. Recursos Naturales y Ambiente 43, 90-96. Disponible en: http://web.catie.ac.cr/informacion/RFCA/rev43/pag90-96.pdf

Orozco, J. (1996). Estado del arte: La dimensión ambiental en la investigación en Centroamérica: Costa Rica, Nicaragua y El Salvador. SUDESCA Y CINPE. Recuperado de http://www. cinpe.una.ac.cr/investigacion/nucleos-tematicos/proyectos/sudesca/espanol/publicaciones/ publicaciones-sudesca.html

Palma, J. M. (2005, enero-abril). Los árboles en la ganadería del trópico seco. Avances en investigación agropecuaria, 9(1), 1-11. Recuperado de http://redalyc.uaemex.mx/pdf/837/83709101.pdf 
Pérez, J. I. J., Ramírez , J. F., Monroy, J. F., Campos, J. (2006, noviembre). Ambiente, sociedad, cultura y ambiental en el Estado de México Revista Iberoamericana de Educación 40(4),1-10. Recuperado de http://www.rieoei.org/deloslectores/1581Perez.pdf

Pérez, V. M. (1977). Veinticinco años de investigación sistemática del cultivo del café en Costa Rica: 1950-1975. Agronomía Costarricense, 1(2), 169-185.

Quesada, G. (2008). Garantías ambientales: un nuevo modelo ecológico-político para Costa Rica y el mundo. Biocenosis 21(1-2), 119-124. Recuperado de http://web.uned.ac.cr/biocenosis/ images/stories/articulosVol21/Biocenosis21 22.pdf

Román, M. (2007). Desarrollo turístico e inmobiliario costero y preocupaciones ambientales. Decimotercer informe sobre el estado de la nación en desarrollo humano sostenible (Informe final). Recuperado de http://unpan1.un.org/intradoc/groups/public/documents/icap/ unpan030197.pdf

Rosero-Bixby, L. y Palloni, A. (1998). Población y deforestación en Costa Rica. En Conservación del bosque en Costa Rica (pp. 131-150). San José, Costa Rica : Academia Nacional de Ciencias y Programa Centroamericano de Población. Recuperado de http://ccp.ucr.ac.cr/bvp/ pdf/bosques/rosero47.pdf

Sánchez-Azofeifa, G. A., Kalacska, M., Quesada, M., Stoner, K., Lobo, J. A. y Arroyo-Mora, J. P. (2003). Tropical dry climates. En: M. D. Schwartz (Ed.), Phenology: An Integrative Environmental Science (pp. 121-137). The Netherlands: Kluwer Academic Press.

Valerio, C. E. (1991). La diversidad biológica de Costa Rica. San José, Costa Rica: Editorial Heliconia.

Vargas, E. (s. f. a). Impactos ambientales de los desarrollos turísticos y residenciales Identificación y análisis de impactos ambientales relevantes de los desarrollos turísticos y residenciales en la costa pacífica de Costa Rica (Informe final). Recuperado de http://www.responsibletravel. org/resources/documents/Coastal-tourism-documents/Informe E Vargas Enviro Impact CREST Abril 2009\%5B1\%5D.pdf

Vargas, R. (2009 b). Mientras RIU se ríe de nuestra legislación ambiental, el pueblo abre en sus narices un nue. Kioscos Ambientales UCR. Recuperado de http://kioscosambientales.ucr. ac.cr/index.php?option $=$ com $\_$content $\& v i e w=$ article $\& i d=143$ :mientras-riu-se-rie-de-nuestralegislacion-ambiental-el-pueblo-abre-en-sus-narices-un-nue \&catid=46:opinion $\&$ Itemid=67

Zamora-Trejos, P. y Cortes, J. (2009, septiembre). Los manglares de Costa Rica: el Pacífico Norte. Revista de Biología Tropical, 57(3), 473-488 